\title{
Nanophotonic Modulator with Bismuth Ferrite as Low-loss Switchable Material
}

\author{
Babicheva, Viktoriia; Zhukovsky, Sergei; Lavrinenko, Andrei
}

Published in:

Proceedings of 2015 Conference on Lasers and Electro-Optics (CLEO)

Link to article, DOI:

10.1364/cleo_at.2015.jtu5a.72

Publication date:

2015

Document Version

Peer reviewed version

Link back to DTU Orbit

Citation (APA):

Babicheva, V., Zhukovsky, S., \& Lavrinenko, A. (2015). Nanophotonic Modulator with Bismuth Ferrite as Lowloss Switchable Material. In Proceedings of 2015 Conference on Lasers and Electro-Optics (CLEO) IEEE. https://doi.org/10.1364/cleo_at.2015.jtu5a.72

\section{General rights}

Copyright and moral rights for the publications made accessible in the public portal are retained by the authors and/or other copyright owners and it is a condition of accessing publications that users recognise and abide by the legal requirements associated with these rights.

- Users may download and print one copy of any publication from the public portal for the purpose of private study or research.

- You may not further distribute the material or use it for any profit-making activity or commercial gain

- You may freely distribute the URL identifying the publication in the public portal 


\title{
Nanophotonic Modulator with Bismuth Ferrite as Low-loss Switchable Material
}

\author{
Viktoriia E. Babicheva, ${ }^{1,2,}$ ' Sergei V. Zhukovsky, ${ }^{1,2}$ and Andrei V. Lavrinenko ${ }^{1}$ \\ ${ }^{I}$ DTU Fotonik, Technical University of Denmark, Oersteds Plads 343,2800 Kgs. Lyngby, Denmark \\ ${ }^{2}$ ITMO University, Kronverkskiy, 49, St. Petersburg 197101, Russia \\ *v.babicheva@phoi.ifmo.ru
}

\begin{abstract}
We propose a nanophotonic waveguide modulator with bismuth ferrite as a tunable material. Due to near-zero losses in bismuth ferrite, modulation with up to $20 \mathrm{~dB} / \mu \mathrm{m}$ extinction ratio and $12 \mu \mathrm{m}$ propagation length is achieved.

OCIS codes: (240.6680) Surface plasmons; (250.7360) Waveguide modulators
\end{abstract}

\section{Introduction}

Nanophotonic and plasmonic modulators and switches are of major interest for ultra-compact integrated circuits and have been extensively studied for the last several years. Potentially promising designs have been proposed using various tunable materials, such as silicon [1], transparent conductive oxides (TCOs) [2-4], nonlinear polymers [5], and vanadium dioxide [6]. Silicon and TCOs, where switching occurs due to carrier concentration mechanisms, provide the highest bit rate. The other considered materials, where switching occurs due to structural transformations within the material, are slower but feature much deeper refractive index modulation, decreasing the device footprint.

One example of the latter family of materials are ferroelectric materials, such as bismuth ferrite $\left(\mathrm{BiFeO}_{3}, \mathrm{BFO}\right)$ or barium titanate $\left(\mathrm{BaTiO}_{3}, \mathrm{BTO}\right)$, where refractive index can be changed by applying voltage to partially reorient the ferroelectric domains. BTO was shown to provide effective performance in photonic thin film modulators $[7,8]$. Recently it was shown that BFO has refractive index difference $\Delta n_{\mathrm{BFO}}=0.18$, which is about three times higher birefringence than BTO [9], This strong birefringence has been proposed for electro-optic modulation [10,11].

Here, we study an implementation of BFO as switchable material for plasmonic waveguide modulators. The device schematic is shown in Fig. 1(a). We focus on the modulator design based on metal-insulator-metal (MIM) waveguide configuration, and characterize the performance of these modulators.

(a)

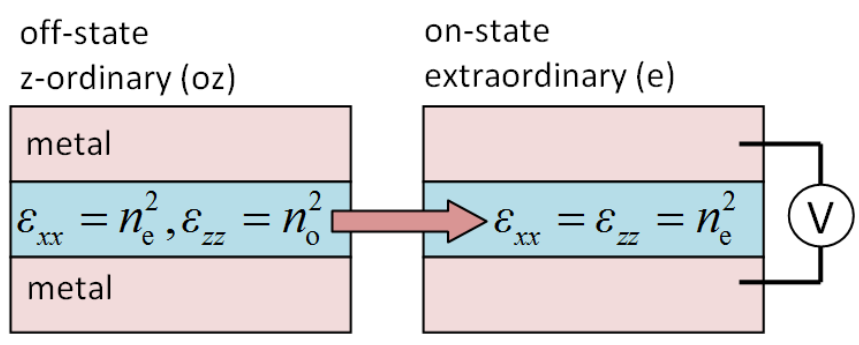

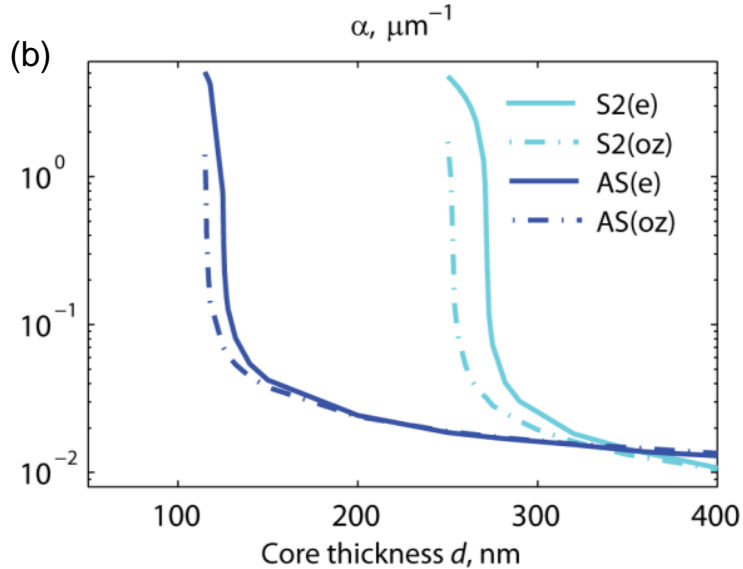

Fig. 1. (a) Schematic view of the proposed plasmonic modulator based on metal-insulator-metal waveguide with the switchable BFO core in the off-state (left) and the on-state (right). (b) Calculated absorption coefficients for two different modes of the MIM waveguide: symmetric S2 and antisymmetric AS in the off- and on-state. Labels (oz) and (e) denote the off-state (z-ordinary) and the on-state, respectively.

\section{Modulator concept and performance}

To characterize the action of the modulator, we begin by determining the plasmonic modes of an MIM waveguide shown in Fig. 1(a) with birefringent BFO core, for which $n_{\mathrm{o}}=2.83$ and $n_{\mathrm{e}}=2.65$ [9]. For the device off-state, we assume $\varepsilon_{x x}=n_{\mathrm{e}}^{2}$ and $\varepsilon_{z z}=n_{\mathrm{o}}^{2}$ ("z-ordinary", denoted "oz"), and the imaginary part of $\varepsilon_{i i}$ is assumed to be negligibly small at $1.55 \mu \mathrm{m}$. Under applied voltage, the domains are reoriented, and the off-state switches to the on-state (labeled "e") with permittivity tensor components equal to $\varepsilon_{x x}=\varepsilon_{z z}=n_{\mathrm{e}}^{2}$. Hence there should be a change in the parameters of the guided modes when the device switches between the off- and on-state. Two modes, one symmetric (labeled S2) and one antisymmetric (labeled AS) have been selected for analysis, with the calculated dependence of 
mode absorption coefficients on the core thickness shown in Fig. 1(b). We see that the both modes S2 and AS feature a cut-off in the MIM waveguide. In accordance with expectations, the cut-offs occur for slightly different core thickness for the off-state vs. the on-state. Hence, amplitude modulation of light in the guided modes can be realized by applying the modulated voltage to the MIM waveguide claddings, also serving as electrodes.

To compare the modulator performance, one can define the figure of merit (FoM) of the device as $F=\left(\alpha_{\mathrm{e}}-\alpha_{\mathrm{o}}\right) / \alpha_{\mathrm{o}}$. Figure 2(a) shows that both considered modes have high FoMs in the cut-off region, where there is an abrupt $\alpha$ change. For the symmetric mode S2, the FoM value can reach 67 . At this point, the extinction ratio $E=8.68\left(\alpha_{\mathrm{e}}-\alpha_{\mathrm{o}}\right)=20 \mathrm{~dB} / \mu \mathrm{m}$, and consequently $3 \mathrm{~dB}$ switch can be realized on the $150 \mathrm{~nm}$ distance. Corresponding propagation length $z=1 /\left(2 \alpha_{\mathrm{o}}\right)$ is $12 \mu \mathrm{m}$, as shown in Fig. 2(b). For the asymmetric mode AS, the device characteristics are also prominent: FoM up to 29 , extinction ratio $E=28 \mathrm{~dB} / \mu \mathrm{m}$ (allowing a $3 \mathrm{~dB}$ switch on $107 \mathrm{~nm}$ ), and propagation length $z_{\mathrm{AS}}=3.5 \mu \mathrm{m}$ (see Fig. 2).

(a)

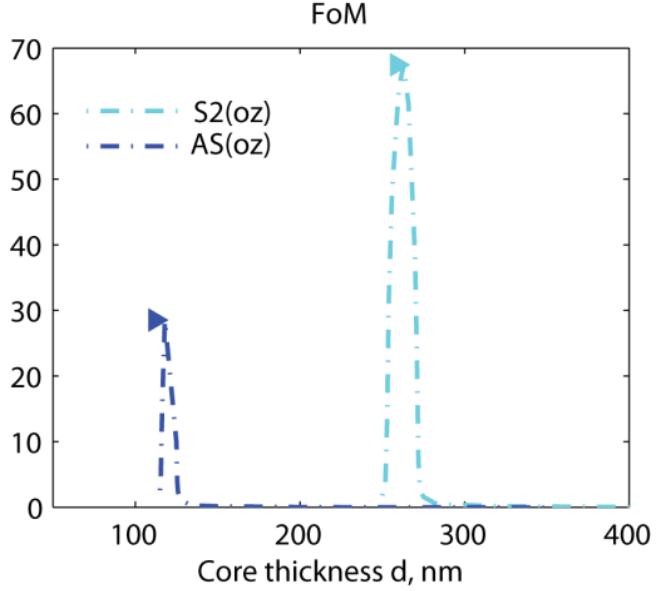

(b)

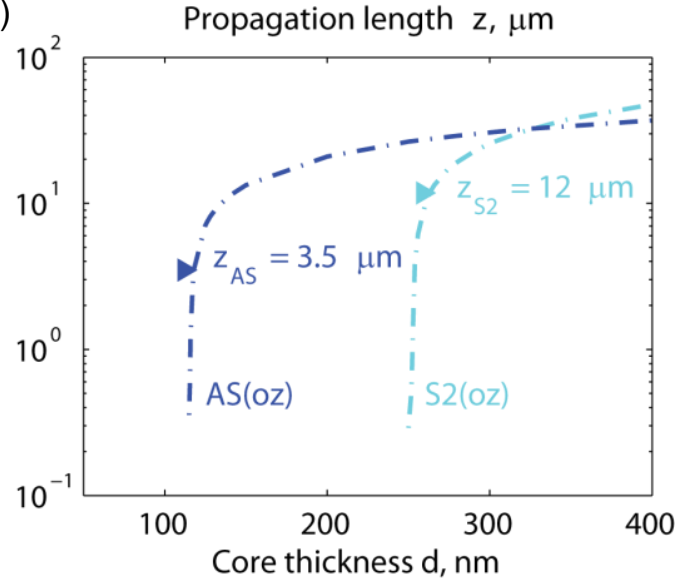

Fig. 2. (a) FoM and (b) propagation length in the less lossy off-state $z$ for the second symmetric (S2) and asymmetric (AS) modes. Triangular marks in (b) correspond to maxima of the FoM in (a).

\section{Discussion and conclusions}

The proposed designs possess the following advantages. Low losses of BFO do not cause additional attenuation in the waveguide core, and thus do not increase insertion loss of the whole device, in contrast to TCO or vanadium dioxide. The MIM configuration allows cut-off of the propagating mode and thus makes it possible to modulate propagation signal by switching it on and off with the metal layers serving as electrodes. In such a waveguide, the mode is highly confined within the core and the field does not expand outside waveguide cladding.

Our calculations show that effective signal amplitude control by direct change of mode absorption coefficient can be realized. In this case a BFO-based plasmonic modulator can have FoM up to 67, which is much higher than in previously reported devices based on indium tin oxide, vanadium dioxide, or InGaAsP active layers [2,6,12,13].

\section{References}

[1] J. A. Dionne et al, "PlasMOStor: a metal-oxide-Si field effect plasmonic modulator," Nano Lett. 9, 897 (2009).

[2] V. E. Babicheva and A. V. Lavrinenko, "Plasmonic modulator optimized by patterning of active layer and tuning permittivity," Opt. Commun. 285, 5500 (2012).

[3] V. J. Sorger et al, "Ultra-compact silicon nanophotonic modulator with broadband response," Nanophotonics 1, 17 (2012).

[4] V. E. Babicheva et al, "Towards CMOS-compatible nanophotonics: Ultra-compact modulators using alternative plasmonic materials," Opt. Express 21, 27326 (2013).

[5] A. Melikyan et al, "High-speed plasmonic phase modulators," Nature Photonics 8, 229 (2014).

[6] B.A. Kruger et al, "Design of electrically driven hybrid vanadium dioxide (VO2) plasmonic switches," Opt. Express 20, 23598 (2012).

[7] M. J. Dicken et al, "Electrooptic modulation in thin film barium titanate plasmonic interferometers," Nano Lett. 8, 4048 (2008).

[8] V. E. Babicheva, A. V. Lavrinenko, "Plasmonic modulator based on metal-insulator-metal waveguide with barium titanate core", Photonics Letters of Poland 5, 57 (2013).

[9] S.G. Choi et al, "Optical anisotropy and charge-transfer transition energies in BiFeO3 from 1.0 to 5.5 eV," Phys. Rev. B. 83, 100101(R) (2010). [10] S. H. Chu, D. J. Singh, J. Wang, E.-P. Li, K. P. Ong, "High optical performance and practicality of active plasmonic devices based on rhombohedral BiFeO3," Laser \& Photonics Reviews 6, 684 (2012).

[11] V. E. Babicheva, S. V. Zhukovsky, and A.V. Lavrinenko, "Bismuth ferrite as low-loss switchable material for plasmonic waveguide modulator," Opt. Express 22, 28890 (2014).

[12] V.E. Babicheva et al, "Plasmonic modulator based on gain-assisted metal-semiconductor-metal waveguide," Photon. Nanostructures 10, 389 (2012). [13] V. E. Babicheva, R. Malureanu, and A. V. Lavrinenko, "Plasmonic finite-thickness metal-semiconductor-metal waveguide as ultra-compact modulator," Photon. Nanostructures 11, 323 (2013). 